\title{
The stellar population of the Rosat North Ecliptic Pole survey
}

\section{Spectral analysis}

\author{
L. Affer ${ }^{1,2}$, G. Micela ${ }^{1}$, and T. Morel ${ }^{3}$ \\ 1 Istituto Nazionale di Astrofisica, Osservatorio Astronomico di Palermo G. S. Vaiana, Piazza del Parlamento 1, 90134 Palermo, Italy \\ e-mail: affer@astropa.inaf.it \\ 2 Dipartimento di Scienze Fisiche ed Astronomiche - Università di Palermo, Piazza del Parlamento 1, 90134 Palermo, Italy \\ 3 Katholieke Universiteit Leuven, Departement Natuurkunde en Sterrenkunde, Instituut voor Sterrenkunde, Celestijnenlaan 200D, \\ 3001 Leuven, Belgium
}

Received 10 January 2008 / Accepted 16 February 2008

\begin{abstract}
Context. X-ray surveys allow to identify young, main-sequence stars in the solar neighborhood. Young, stellar samples, selected according to their activity, can be used to determine the stellar birthrate in the last billion years. The ROSAT North Ecliptic Pole survey (NEP), with its moderately deep sensitivity (fluxes $\approx 10^{-14} \mathrm{erg} \mathrm{cm}^{-2} \mathrm{~s}^{-1}$ ), is the best survey, to date, able to sample the intermediate-age $\left(10^{8}-10^{9}\right.$ years) nearby population. The identification process of NEP X-ray sources resulted in 144 X-ray sources having a normal stellar counterpart, with an excess of yellow stars with respect to model predictions.

Aims. We want to determine if these X-ray active stars are young or intermediate-age stars, or active binaries.

Methods. We acquired high-resolution, high signal-to-noise ratio optical spectra, to determine the age and physical properties of the NEP X-ray-detected stellar sources. We measure the (i) lithium abundance using the Li I $6707.8 \AA$ line, which is an excellent, youth indicator for our age range of interest; (ii) rotational and radial velocities (through cross-correlation methods); and (iii) chromospheric emission (from $\mathrm{H} \alpha$ and $\mathrm{Na}$ I $D_{1}$ and $D_{2}$ lines).

Results. The radial velocities distribution is consistent with that of a young field star population of age $4 \times 10^{9}$ yrs, or younger. Rotational velocity measurements imply that our sample is dominated by relatively young or intermediate-age stars, as confirmed by our lithium measurements.

Conclusions. Most of the detected stars probably belong to a young or intermediate-age population. Our measurements suggest that a burst in the stellar birthrate of a factor of four occurred in the last $10^{8}$ years. We cannot, however, exclude the possibility that a small fraction of sources, amongst the fastest of the K-rotators, are old binary systems with tidally-locked rotation.
\end{abstract}

Key words. stars: rotation - stars: abundances - techniques: spectroscopic - techniques: radial velocities -

Galaxy: solar neighbourhood

\section{Introduction}

The analysis of the stellar component of flux-limited X-ray surveys is a powerful tool in the study of the young stellar population of the Galaxy. Optical data, which helps determine photospheric parameters, cannot alone discriminate between mainsequence stars of different age. In contrast, the average X-ray luminosity of normal, late-type stars decreases by up to 3 orders of magnitude mainly during the main-sequence lifetime (Micela et al. 1985, 1988; Guedel et al. 1997; Feigelson \& Montmerle 1999); optical properties, however, remain almost unchanged. Stellar X-ray surveys allow to study in detail the global properties of stellar populations in the solar neighborhood, in particular of the young population, since young stars are detected, in X-rays, out to larger distances than older stars. Shallow stellar, flux-limited, X-ray selected samples will be dominated by young stars; in contrast, deep high-latitude stellar X-ray samples will be dominated by old stars, because young stars have smaller scale height, and deep X-ray surveys probe distances beyond the scale height of the youngest population (Micela et al. 2001). The comparison between observations and predictions from galactic models offers an important tool to constrain the spatial distribution of young stellar population and to infer the local stellar birthrate in the last billion years.
Analyses of both the Einstein Extended Medium Sensitivity Survey (EMSS, Gioia et al. 1990), and XMM-Newton Bright Serendipitous Survey (XBSS, Della Ceca et al. 2004) measured an excess of yellow stars (Sciortino et al. 1995; Lopez-Santiago et al. 2006, respectively). For the EMSS, these yellow stars appear to be young, in agreement with their measurement of lithium abundance (Favata et al. 1993). For the XBSS, the excess may represent a young population, or a number of active binary systems that have a yellow dwarf as a primary (Lopez-Santiago et al. 2007).

The study of the stellar content of the ROSAT North Ecliptic Pole survey (NEP hereafter, Henry et al. 2001) confirmed the existence of an excess of yellow stars in the solar neighborhood even at intermediate X-ray flux, which is probably due to a young stellar population (Micela et al. 2007, hereafter referred to as Paper I).

The NEP survey is a survey of X-ray sources, covering a $9^{\circ} \times 9^{\circ}$ area centred on the Galactic North Ecliptic Pole $\left(l=96^{\circ} .4\right.$, $b=29.8)$. This is the sky region with the highest sensitivity observed during the ROSAT All Sky Survey (RASS), with a total exposure time amounting to $\sim 40 \mathrm{ks}$ at the pole. The relatively high galactic latitude, together with the moderate sensitivity, permits to observe young stars close to their scale height. 
An overview of the NEP survey, including both its selection function and optical identification program, can be found in Henry et al. (2001); Voges et al. (2001) summarized the X-ray data and source statistical properties; Gioia et al. (2001) provided evidence for cluster X-ray luminosity evolution, and Mullis et al. (2001) described the NEP supercluster discovered in the survey X-ray data. The extragalactic component of the NEP survey was discussed in Mullis et al. (2003, 2004a,b), Gioia et al. (2004), and Henry et al. (2006).

The identification of the optical counterparts (based both on optical photometry and spectroscopy of all candidate counterparts) to each of the X-ray sources was completed by Gioia et al. (2003). This X-ray based data set is unique in its combination of contiguous sky coverage $\left(80 \mathrm{deg}^{2}\right)$, and sensitivity, which is higher than any of the Einstein-based surveys.

More recent X-ray surveys, such as the Chandra Deep Fields, are far deeper than the NEP survey, but have small sky coverage. These surveys are biased towards older stars, which are more numerous but fainter in X-rays, with the younger stellar population being very poorly sampled. The small sky coverage does not, however, allow robust conclusions to be made.

The stellar content of the Chandra deep survey (Feigelson et al. 2004) reveals that the old star population does not contain any excess of yellow stars, but instead a deficiency of $\mathrm{dG}-\mathrm{dK}$ stars, while the signatures of $\mathrm{dM}$ stars are reproduced perfectly by galactic models. This behaviour is the opposite of that observed for the young stellar population, and suggests that some changes occur at intermediate ages, where NEP survey may give a unique contribution thanks to its intermediate sensitivity. Thus the NEP survey is unique in its ability to properly sample the young and intermediate-age stellar populations in the Galactic disk.

In Paper I we analyzed the stellar content of the NEP survey, to determine the nature of active stars in the solar neighborhood. We determined the spectral types for all source detections. We then compared the observational data with the predictions of the XCOUNT model (Favata et al. 1992; Micela et al. 1993), and, at intermediate X-ray fluxes, ascertained the presence of an excess of active yellow stars, in the solar neighborhood.

To characterize the stellar population of the NEP survey and determine the nature of the observed excess, we derived, in the second paper of this series, the physical properties of the X-ray sources, such as radial and rotational velocities, and lithium abundances. In a forthcoming paper, we will determine the chemical composition of the stellar photospheres; this will allow the study of the enrichment history of the interstellar matter and, combined with kinematical data and ages, will provide a powerful way of probing the chemical and dynamical evolution of the Galaxy.

The present paper is organized as follows: in Sects. 2 and 3, we describe the selection rules of our sample, the observations, and the data reduction. In Sect. 4, we describe the methods of analysis and discuss the distribution of radial and rotational velocities, while binary stars and lithium-abundance determination are described in Sects. 5 and 6. In Sects. 7 and 8, we discuss and summarize our findings.

\section{Observations and data reduction}

To complete our proposed investigation, we would have ideally wanted to observe the entire sample of NEP stars. Due to telescope and instrumentation constraints we instead had to restrict our sample to stars with $J \leq 11$ in the 2MASS Catalogue; this selection criterion should not affect the achievement of our goals,
Table 1. Sampled stars per spectral type.

\begin{tabular}{rcc}
\hline \hline Sp.type & N.Obs.(Paper I) & N.Obs.(This work) \\
\hline A & 3 & 1 \\
F0-F5 & 10 & 9 \\
F6-F9 & 8 & 4 \\
G & 29 & 18 \\
K & 53 & 22 \\
M & 41 & 2 \\
\hline Total & 144 & 56 \\
\hline
\end{tabular}

apart from the study of dM stars. In Table 1, we summarize the spectral type classification of stellar X-ray NEP sources, as derived in Paper I, together with the sample observed in the present work. As can be seen from Table 1, we covered almost the entire sample of early-F (F0-F5) stars, while we sampled a fraction between $40 \%$ and $60 \%$ of late-F to $\mathrm{G}$ and $\mathrm{K}$ stars; dM stars were poorly sampled (due to their faintness at the energies considered) and we are unable to measure reliably their mean properties, even though, with $\mathrm{dK}$ stars, they form a substantial part of the data set. Finally we can state that our sample is representative of stars earlier than dM.

The observations, with signal-to-noise ratios between 40 and 440, were obtained (in service mode) during several observing runs between January and June 2004 (see Table A.1) at the Telescopio Nazionale Galileo (TNG) located at the Observatorio del Roque de Los Muchachos (La Palma, Canary Islands).

Our survey contains 56 stars in the region of sky of $80.6 \mathrm{deg}^{2}$ about the North Ecliptic Pole (NEP) at $\alpha=18^{\mathrm{h}} 00^{\mathrm{m}} 00^{\mathrm{s}}, \delta=$ $+66^{\circ} 33^{\prime} 00^{\prime \prime}$ (J2000.0), at a Galactic latitude, $b=29.8$, with spectral types in the range $\mathrm{F} 0 \mathrm{~V}-\mathrm{M} 3 \mathrm{~V}$, and values of $B-V$ in the range 0.3 to 1.5 , which corresponds approximately to effective temperatures of about $7300 \mathrm{~K}-3500 \mathrm{~K}$. For all observations, we used the cross-dispersed echelle SARG spectrograph attached to the $3.58 \mathrm{~m}$ TNG. The dioptric camera has a focal ratio of $f / 4.8$, and the detector is a mosaic of $22 \mathrm{k} \times 4 \mathrm{k}$ thinned and back illuminated CCDs, with a $13.5 \mu \mathrm{m}$ pixel size, and a scale of $0.16 \mathrm{arcsec} / \mathrm{pixel}$. We used the Yellow grism, with a dispersion of $61.0 \AA / \mathrm{mm}$, the FW4 filter, and selected a binning of $2 \times 1$. The spectra continuously cover a wavelength range from $4620 \AA$ to $7920 \AA$, with a resolving power of about 57000 , yielding a FWHM of $5 \mathrm{~km} \mathrm{~s}^{-1}$.

To enable a differential analysis, we obtained a solar flux spectrum, i.e. a high $S / N(\approx 500)$ moonlight spectrum acquired using the same instrumental configuration as the target stars.

As preparation for our cross-correlation analysis, the objective of the data reduction is to produce wavelength-calibrated, one-dimensional spectra for each echelle order, free of relevant instrumental and telluric signatures. Tasks, implemented in the IRAF ${ }^{1}$ package, were used to carry out the standard reduction procedures on echelle spectra (i.e. bias subtraction, flatfield correction, removal of scattered light, order extraction, and wavelength calibration). The two CCDs were separated and reduced independently. Numerous emission spikes, possibly arising from CCD electronics, were initially found to affect the extracted spectra. To remedy this problem, the data were extracted after excluding deviant pixels in the two-dimensional science frames. The rejection thresholds of the sigma-clipping algorithm were varied to remove the artefacts efficiently, without affecting

\footnotetext{
1 IRAF (Image Reduction and Analysis Facility) is distributed by the National Optical Astronomy Observatories, operated by the Association of Universities for Research in Astronomy, Inc., under cooperative agreement with the National Science Foundation.
} 
significantly the remainder of the spectrum. The wavelength calibration was performed using Thorium-Argon lamp exposures acquired each night. For three nights, Thorium-Argon lamp exposures were not acquired; for data obtained during this time, wavelength calibration was completed using lamp exposures available for the night closest in time; we identify the spectra affected by labelling the corresponding stars with an asterisk in Table A.1 (we use a similar symbol in all tables). We compared the wavelength shifts between telluric lines of these spectra, and of spectra for which wavelength calibration was completed using lamp data acquired during the same night; we corrected each spectrum by the corresponding wavelength shift (which amounts, at most, to $0.9 \mathrm{~km} \mathrm{~s}^{-1}$ ).

\section{Analysis methods and results: radial and rotational velocities}

In Paper I, we analyzed the stellar content of the NEP survey and we determined spectral types of all of the stellar counterparts to the 144 ROSAT NEP X-ray sources. Our analysis confirmed the presence of an excess of active yellow stars, in agreement with previous results for shallower surveys. To characterize the nature of the NEP stellar sample, we determine the radial velocity $(R V)$ of each star, which provides a kinematic index of the population to which they belong, and the projected rotational velocity $v \sin i$, which is strongly correlated with the X-ray luminosity in single stars (Pallavicini et al. 1981), and evolves with stellar age (Skumanich 1972).

We derive simultaneously the radial and rotational velocities using the Fourier Cross-correlation technique originally described by Tonry \& Davis (1979). The cross-correlation technique was developed to derive robust radial velocities using all of the information in the spectrum at once, rather than one spectral line at a time. We correlate the spectrum of a program star against that of a stellar template. The position of the correlation peak indicates the relative radial velocity, and the breadth of the peak is interpreted as being due to rotational broadening.

\subsection{Radial velocities}

To check the robustness of our approach, we compared the crosscorrelation method with that performed line by line, then we calculated radial velocities adopting three different approaches which rely on:

1. Wavelength shifts of selected photospheric absorption features.

2. Cross-correlation function of the object spectrum with that of a template star belonging to the sample.

3. Cross-correlation function of the object spectrum with that of the Sun, obtained with the same instrumental configuration as the target stars.

We comment on the application of all three methods:

1. The heliocentric $R V \mathrm{~s}$ of the sample stars were obtained by measuring the wavelength shifts of a set of 58 measurable and unblended prominent photospheric absorption features (Table 2), using the IRAF task RVIDLINES, and adopting the weighted mean of the results (using the inverse of the variances as weights). The variance of the weighted mean is the inverse of the sum of the weights.

2. We calculated the $R V$ of the sample stars using the IRAF task FXCOR, which performs a Fourier Cross-Correlation of
Table 2. Absorption features selected to calculate radial velocities from wavelength shifts.

\begin{tabular}{|c|c|c|c|}
\hline Wavelength $(\AA)$ & Element & Wavelength $(\AA)$ & Element \\
\hline 6419.95 & Fe I & 6705.10 & $\mathrm{Fe} \mathrm{I}$ \\
\hline 6421.35 & $\mathrm{Fe} I$ & 6710.32 & $\mathrm{Fe} I$ \\
\hline 6430.85 & $\mathrm{Fe} I$ & 6717.69 & $\mathrm{Ca}$ \\
\hline 6439.07 & $\mathrm{Ca} \mathrm{I}$ & 6726.67 & $\mathrm{Fe} I$ \\
\hline 6449.81 & $\mathrm{Ca} \mathrm{I}$ & 6743.12 & Ti I \\
\hline 6450.23 & CoI & 6750.15 & $\mathrm{Fe} I$ \\
\hline 6455.60 & $\mathrm{Ca} \mathrm{I}$ & 6767.77 & $\mathrm{Ni}$ I \\
\hline 6462.57 & $\mathrm{Ca} \mathrm{I}$ & 6783.70 & $\mathrm{Fe} I$ \\
\hline 6469.19 & $\mathrm{Fe} \mathrm{I}$ & 6786.86 & $\mathrm{Fe} I$ \\
\hline 6471.66 & $\mathrm{Ca} I$ & 6806.84 & $\mathrm{Fe} I$ \\
\hline 6475.62 & $\mathrm{Fe} I$ & 6810.26 & $\mathrm{Fe} \mathrm{I}$ \\
\hline 6481.87 & $\mathrm{Fe} I$ & 6814.95 & Co I \\
\hline 6482.80 & Ni I & 6820.37 & $\mathrm{Fe} \mathrm{I}$ \\
\hline 6493.78 & $\mathrm{Ca} \mathrm{I}$ & 6828.59 & $\mathrm{Fe} I$ \\
\hline 6494.98 & Fe I & 6839.83 & $\mathrm{Fe} I$ \\
\hline 6495.74 & Fe I & 6841.34 & $\mathrm{Fe} I$ \\
\hline 6496.47 & $\mathrm{Fe} I$ & 6842.04 & $\mathrm{Ni}$ I \\
\hline 6496.90 & Ba II & 6842.69 & $\mathrm{Fe} I$ \\
\hline 6569.22 & $\mathrm{Fe} \mathrm{I}$ & 6843.66 & $\mathrm{Fe} I$ \\
\hline 6572.78 & $\mathrm{Ca} \mathrm{I}$ & 7090.38 & $\mathrm{Fe} I$ \\
\hline 6574.23 & Fe I & 7095.43 & $\mathrm{Fe} I$ \\
\hline 6575.02 & $\mathrm{Fe} I$ & 7110.90 & $\mathrm{Ni}$ I \\
\hline 6581.21 & $\mathrm{Fe} I$ & 7112.18 & $\mathrm{Fe} I$ \\
\hline 6586.31 & $\mathrm{Ni} \mathrm{I}$ & 7122.20 & $\mathrm{Ni}$ I \\
\hline 6592.91 & $\mathrm{Fe} I$ & 7130.92 & $\mathrm{Fe} I$ \\
\hline 6593.87 & $\mathrm{Fe} \mathrm{I}$ & 7142.52 & $\mathrm{Fe} I$ \\
\hline 6598.60 & $\mathrm{Ni} I$ & 7145.32 & $\mathrm{Fe} I$ \\
\hline 6609.11 & $\mathrm{Fe} I$ & 7148.15 & $\mathrm{CaI}$ \\
\hline 6703.57 & Fe I & 7151.49 & $\mathrm{Fe} I$ \\
\hline
\end{tabular}

a given spectrum with a "template" stellar spectrum, as described by Tonry \& Davis (1979). For each star observed, we cross-correlated its spectrum with that of the template using the wavelength range between about $6625 \AA$ and $6710 \AA$, which contains no significant telluric absorption lines. While performing the cross-correlations for individual stars, the wavelength ranges used were visually inspected and obvious emission lines, cosmic rays, and/or bad pixels were masked. As our stellar template, we used a high signal-tonoise $(S / N \approx 180)$ spectrum of the bright star nep 6163 $(V \approx 7.6)$, which has a modest projected rotational velocity $\left(v \sin i \approx 3.7 \mathrm{~km} \mathrm{~s}^{-1}\right.$, derived by comparison with synthetic spectra computed using ATLAS9). The heliocentric $R V$ of the template object nep 6163 was obtained by measuring the wavelength shifts of a list of prominent, photospheric, absorption features, as for method 1 . The heliocentric $R V$ of each observed object is then calculated by adding the heliocentric $R V$ of the template star, to the $R V$ of each observed star, with respect to the template. The uncertainty in each value of heliocentric velocity was computed by combining (in quadrature) the uncertainties in the heliocentric $R V$ of the template star, and in the relative $R V$ of the object, measured by cross-correlation with the template star.

3. We performed an analysis similar to method 2, using as a stellar template, a high signal-to-noise $(S / N \approx 400)$ solar spectrum, acquired with the same instrumental configuration as the sample stars. Using IRAF task RVCORRECT, we find the heliocentric correction to the observed radial velocity of the solar spectrum, due to the relative motion Sun-Earth. To measure the $R V$ of each observed star, we 


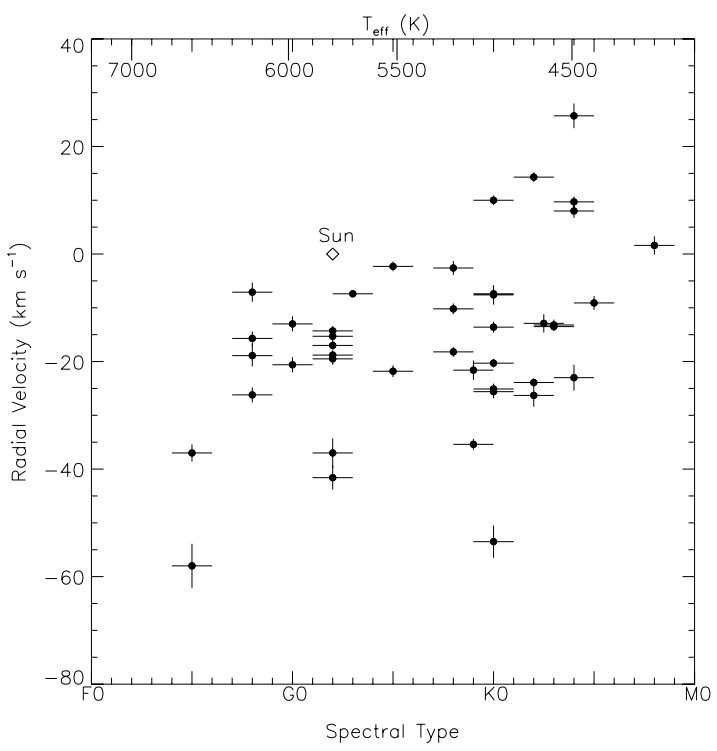

Fig. 1. Heliocentric radial velocity distribution of the NEP sample stars with respect to spectral type (temperature). We assumed that the weighted mean of three independent measures is our estimate of radial velocity for each star. The uncertainty was computed by combining in quadrature the uncertainties of the measures.

cross-correlated each spectrum, with respect to the corrected template spectrum.

The three measurements agree to within a few $\mathrm{km} \mathrm{s}^{-1}$, the maximum difference being about $2.5 \mathrm{~km} \mathrm{~s}^{-1}$; we therefore use the weighted mean, which is obtained using the inverse of the variances as weights, as our radial velocity measurement for each star; the variance that we associate with this weighted mean is the inverse of the sum of the weights.

For a few stars observed, the peak of the cross-correlation function is asymmetric, probably due to binarity and/or high rotation rates $\left(\geq 60 \mathrm{~km} \mathrm{~s}^{-1}\right)$, particularly in early F-type stars; we are unable however to determine whether the stars are binaries and/or fast rotators because our spectra were obtained during a single night. For about 7 stars, this asymmetry is indicative of a binary nature. These stars are discussed later. Figure 1 shows the heliocentric radial velocities as a function of spectral type for the whole sample. The histogram in Fig. 2 provides the radial velocity distribution for single stars, which peaks significantly, with most velocities being in the range between 0 and $-30 \mathrm{~km} \mathrm{~s}^{-1}$, and has a mean value of about $-15 \mathrm{~km} \mathrm{~s}^{-1}$.

\subsection{Rotational velocities}

The cross-correlation function measures the projected rotational velocities $(v \sin i)$ of our targets, relative to stellar spectra of essentially zero rotational velocity (our "templates"); this is possible because the measured width of the cross-correlation peak is a function of the broadening of the spectrum lines, due to rotation velocity. Assuming that the line broadening of the NEP stellar spectra is dominated by rotational broadening, and that our stellar template is an intrinsically narrow-lined spectrum, the width of the cross-correlation peak is a measure of the $v \sin i$ of the NEP targets, which may be calibrated as discussed below. We use a standard Local Thermodynamic Equilibrium (LTE) spectral analysis with the latest version of the spectral-line analysis code MOOG (Sneden 1973), and a grid of ATLAS9 model

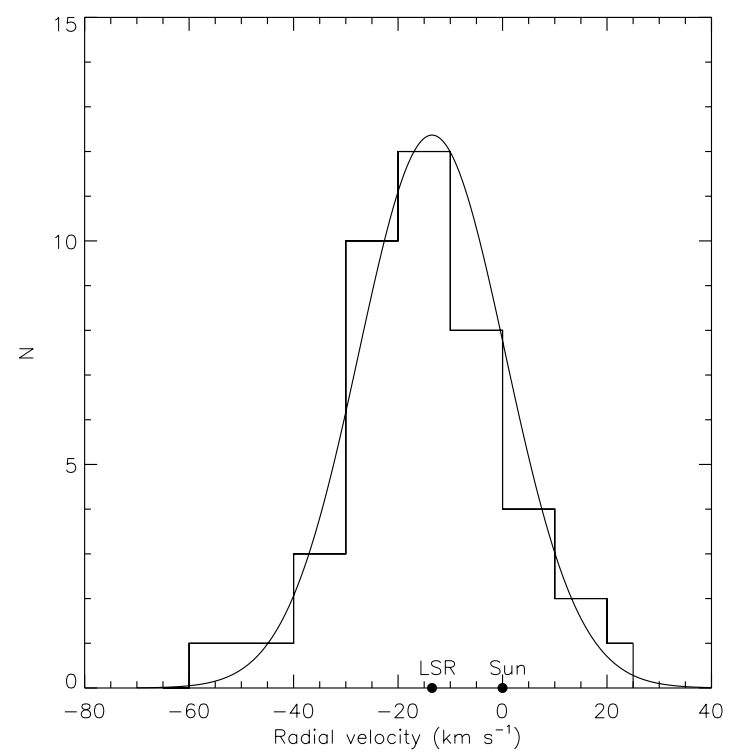

Fig. 2. Histogram of the heliocentric radial velocities, the Sun velocity with respect to the Local Standard of Rest (LSR) is indicated $\left(\left|v_{\text {LSR }}\right|=\right.$ $13.4 \mathrm{~km} \mathrm{~s}^{-1}$, Binney \& Merrifield 1998). The superimposed Gaussian radial velocity distribution for the targets with a standard deviation of $13 \mathrm{~km} \mathrm{~s}^{-1}$, is shown.

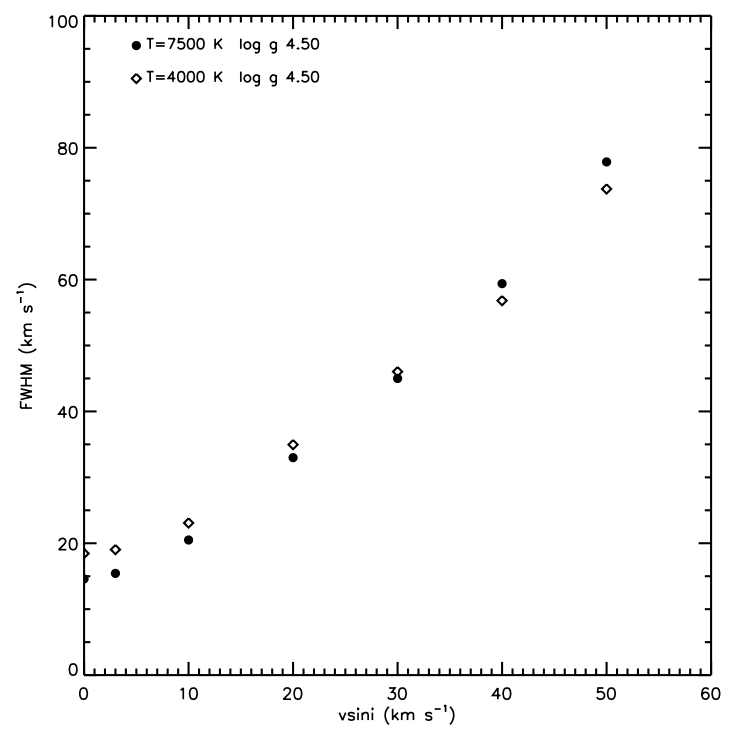

Fig. 3. Calibration curve used for the $v \sin i$ estimation from the width of the cross-correlation peak. The curve was created by cross-correlating the spectrum of the template star with a set of synthetic spectra with different rotational velocities.

atmospheres (Kurucz 1993), to compute synthetic spectra for comparison with the observed spectra.

The ATLAS9 stellar atmospheres are computed without the overshooting option, and by assuming a mixing length to pressure scale height ratio of $\alpha=0.5$. Assumptions made in the models include: the atmosphere is plane-parallel and in hydrostatic equilibrium, the total flux is constant, the source function is described by the Planck function, and the populations of different excitation levels and ionization stages are governed by LTE. The atomic data used to generate synthetic spectra are taken from Kurucz Atomic Line Database (Kurucz 1995).

The cross-correlation is performed with FXCOR. The main peak of the cross-correlation function is fit using a Gaussian function and its FWHM is measured. These FWHM values are 
converted to $v \sin i$ values, using the following calibration process. We compute a grid of synthetic spectra, assuming $\log g=$ $4.5\left(\mathrm{~cm} \mathrm{~s}^{-2}\right)$, microturbulent velocity $\xi=1.0 \mathrm{~km} \mathrm{~s}^{-1}$ and solar metallicity, which are reasonable parameters for our template star, assuming the SARG instrumental resolution, $R=$ 57000 . We convolved these spectra with a rotational broadening function with $v \sin i$ varying from 0 to $50 \mathrm{~km} \mathrm{~s}^{-1}$. We allowed $T_{\text {eff }}$ to vary between $4000 \mathrm{~K}$ and $7500 \mathrm{~K}$ (which are the typical temperature values for our targets) in $500 \mathrm{~K}$ intervals, to check the sensitivity of this relationship to temperature.

The synthetic spectra were cross-correlated with our template and the FWHM of the cross-correlation peak was measured. We compared the results derived for the same analysis using synthetic spectra computed for $\log g=3.0\left(\mathrm{~cm} \mathrm{~s}^{-2}\right)$. The comparison highlights that the calibration results are almost independent of gravity, while the dependence on temperature is more pronounced.

The resulting relation between the FWHM of the crosscorrelation peak and the corresponding $v \sin i$, for the lowest $\left(T_{\text {eff }}=4000 \mathrm{~K}\right)$ and the highest $\left(T_{\text {eff }}=7500 \mathrm{~K}\right)$ temperature values, are shown in Fig. 3.

We note that the discrepancies between the calibration curves, calculated for different $T_{\text {eff }}$, are consistent within the typical errors inferred for the rotational velocities measured for our targets. Using the relationship corresponding to the expected temperature of our template (according to spectral type), we linearly interpolated the FWHM of the cross-correlation peak, with the derived calibration curve to estimate the $v \sin i$ values.

The errors of the rotational velocities were calculated as prescribed by Rhode et al. (2001), to be $v \sin i /(1+r)$, where $r$ is the Tonry \& Davis (1979) parameter, which is a measure of the signal-to-noise ratio of the cross-correlation peak.

When the calibration derived is applied to determine $v \sin i$ for each star, we must pay attention to the minimum value of $v \sin i$ to which this procedure is sensitive. The measured width of the cross-correlation function can be described by the following formula (Tonry \& Davis 1979):

$\sigma_{\text {meas }}^{2}=\sigma_{\text {rot }}^{2}+\sigma_{\text {nat }}^{2}+2 \sigma_{\text {inst }}^{2}$

where $\sigma_{\text {rot }}$ is the rotational width, $\sigma_{\text {nat }}$ is the natural, or intrinsic, line width which is negligible, and $\sigma_{\text {inst }}$ is the instrumental broadening (the last term of the equation includes two contributions because two spectra form the cross-correlation function).

For the derivation of the minimum detectable $v \sin i$, we follow Bailer-Jones (2004). The terms are additive; with perfect data of "infinite" $S / N$ ratio, we could therefore determine "any" rotational broadening, even with non-zero instrumental broadening. In practice, this is not however possible due to noise. BailerJones (2004) assumes that $\sigma_{\text {rot }}$ is detectable only if its value exceeds that of other broadening contributions, and its minimum measurable value is $\sqrt{2} \sigma_{\text {inst }}$. Our theoretical FWHM of the instrumental broadening is fixed by the slit width to be $5 \mathrm{~km} \mathrm{~s}^{-1}$. We note that the full width of a rotational profile corresponds to twice the rotational velocity: one half of the line is created by the blueshifted approaching limb of the star, the other half by the redshifted receding limb (Bailer-Jones 2004). The minimum detectable $v \sin i$ for our data is $\sqrt{2} \times 5.0 / 2=3.5 \mathrm{~km} \mathrm{~s}^{-1}$, which corresponds to the flat portion of the calibrations in Fig. 3.

The cross-correlation technique measures the projected, rotational velocity of the Sun to be $1.63 \pm 0.34 \mathrm{~km} \mathrm{~s}^{-1}$. However, this measurement, which agrees with the well-known value assumed for the Sun $\left(\approx 1.86 \mathrm{~km} \mathrm{~s}^{-1}\right.$, Soderblom 1982), is below the minimum detectable $v \sin i$ of our study. As we can see from

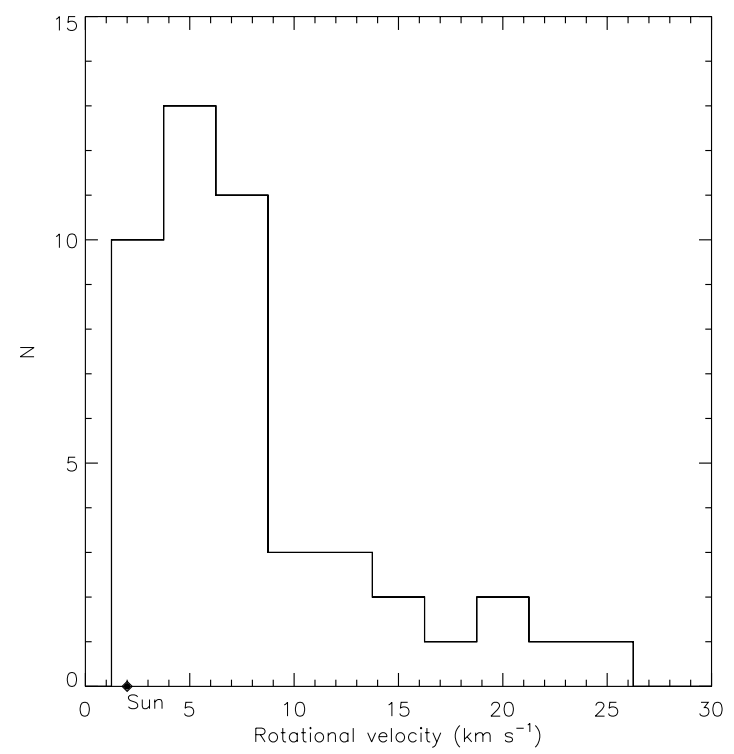

Fig. 4. Histogram of the rotational velocities; almost all of the sample stars have rotational velocities that are comparable or higher than that of the $\operatorname{Sun}\left(\approx 2 \mathrm{~km} \mathrm{~s}^{-1}\right)$.

Fig. 3, this value appears in the flat portion of the calibration relation; the adoption of the mean instrumental broadening as the upper limit on the $v \sin i$ for the slowest rotators, is therefore conservative.

We indicate the $v \sin i$ values derived for slow rotators with $\leq 3.5 \mathrm{~km} \mathrm{~s}^{-1}$.

To perform a further comparison, we adopted a second method to calculate rotational velocities.

We performed a detailed comparison between the observed and synthetic spectra in the wavelength range $6420 \AA-6480 \AA$, which includes several Fe I and Ca I absorption lines, of moderate strength $(\approx 100 \mathrm{~m} \AA)$. Using Kurucz model atmospheres and the MOOG software, we generated a grid of LTE synthetic spectra. We allowed the stellar temperature vary, and assume $\log g=4.5\left(\mathrm{~cm} \mathrm{~s}^{-2}\right), \xi=1.0 \mathrm{~km} \mathrm{~s}^{-1}$, a solar metallicity, and a macroturbulent velocity, that is constrained by the spectral type (Fekel 1997); the rotational velocity was then the only free parameter. Each spectrum was finally convolved by a $0.1 \AA / p x$ Gaussian FWHM, which reproduces the instrumental profile, as estimated by our lamp spectra. For the determination of rotational velocities, we used the neutral iron lines at $6430.85 \AA$, $6546.24 \AA$ and $6569.22 \AA$ and the Ca I lines at $6439.07 \AA$ and $6471.66 \AA$, which were strong but not saturated lines, and unblended with other lines. We compared the observed and synthetic spectra and determined by eye the value of $v \sin i$ that provides the most appropriate fit to the observed target rotational velocity. We assumed that the spread in $v \sin i$ values for individual lines represented the uncertainty in each measurement. We note that the $v \sin i$ values were measured by comparing observed spectra and synthetic profiles that had been convolved by all broadening effects. The minimum value of $v \sin i$ to which this procedure is sensitive is therefore formally zero. The two independent measurements agree to within a few $\mathrm{km} \mathrm{s}^{-1}$; the maximum difference is about $3.1 \mathrm{~km} \mathrm{~s}^{-1}$ (for the low $S / N$ spectra). We therefore calculated the weighted mean of the two measurements of $v \sin i$ and the inverse of the sum of the weights was taken to be the variance associated with the weighted mean. Figure 4 shows the histogram of the rotational velocities. 


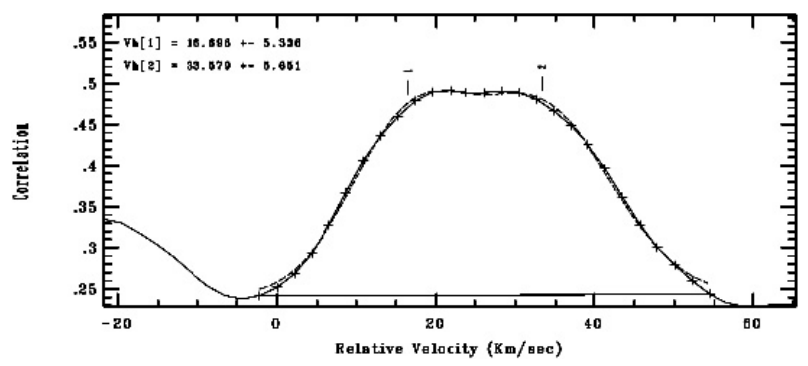

Fig. 5. Example of peaks of the cross-correlation function produced by cross-correlating a binary with our template star. Relative radial velocities of the two components are indicated in the panel.

\section{Binary stars}

For approximately 7 stars, $\approx 12 \%$ of all stars observed, the shape of the FXCOR cross-correlation function peak was asymmetric; this may be indicative of a binary nature for these stars. The peak of the cross-correlation function can be fitted using two overlapping Gaussians, instead of one single function, when the blending between the two stars is not severe; an example of such a case can be seen in Fig. 5. However, we cannot measure the radial velocity of binary systems, because our spectra were acquired during a single night. Both radial and rotational velocities for such stars, when measurable, refer to the contributions of both stars. We report these results for completeness and stress that they are affected by blending of both contributions, and must therefore be taken with extreme caution. The only exception is the spectroscopic binary (SB2) nep 5520, for which spectral lines from both components are clearly separated and the calculations refer separately to each star. For this system, the two components appear to have similar spectral types and effective temperatures, i.e. to contribute equally to the continuum.

\section{Lithium abundances}

Of the 56 total observed stars, 25 show a prominent lithium feature, 5 of which are supposed to be binaries. For these stars, we used the IRAF task Continuum to perform the spectrum normalization. We created a synthetic model atmosphere, using the ATLAS9 code (Kurucz 1993), assuming $\log g=4.5\left(\mathrm{~cm} \mathrm{~s}^{-2}\right)$, $\xi=1.0 \mathrm{~km} \mathrm{~s}^{-1}$ and solar metallicity, and derived the effective temperatures using the star's spectral type.

These models were used to create synthetic spectra for a small interval of approximately $50 \AA$ about the lithium feature at $6707.8 \AA$, from $6700 \AA$ to $6750 \AA$, to determine the line-free regions that were fitted using low-order cubic spline functions, and a variable residual rejection limit. Both the line-free spectral range and the rejection limit were chosen interactively in each case, based on the visual inspection of fitting results. In Fig. 6, we show the normalized spectra about the Li I $6707.8 \AA$ line for two stars observed: the spectrum of nep 3220 does not show the lithium line while nep 4780 shows a prominent line. The Li I $6707.8 \AA$ line is close to the Fe I $6707.44 \AA$ line and both lines are often blended, due to spectral line broadening by stellar rotation. The equivalent width of each of the partiallyblended lines, Li I 6707.8 $\AA$ and Fe I 6707.44 $\AA$, was determined using a two-Gaussian fitting routine provided by IRAF. To estimate uncertainties in the EW measurements, we repeated both the continuum normalization and EW measurement three times. We then accepted the weighted mean of the three measures to be final EW measurements and as typical error the standard

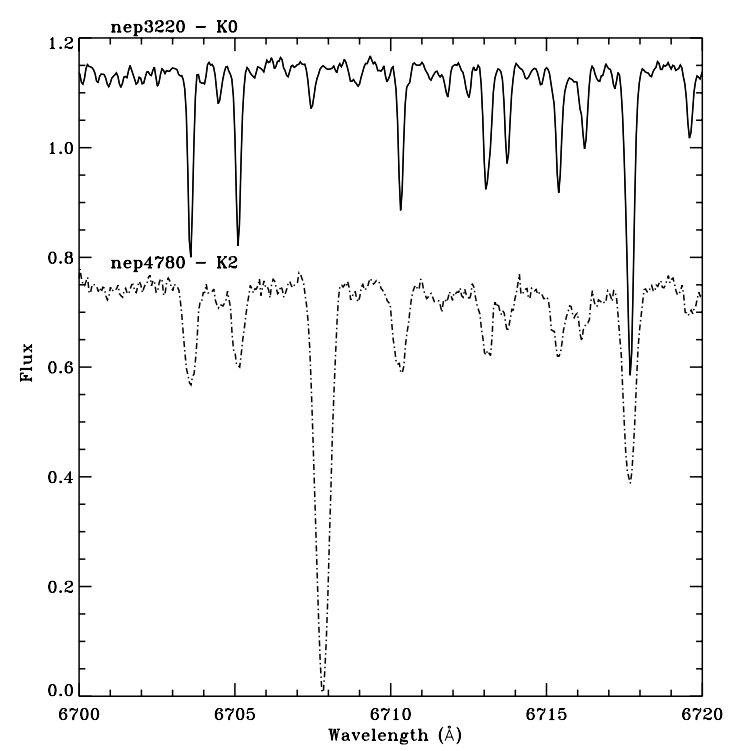

Fig. 6. Spectra in the lithium region for two NEP sources: nep 3220 does not show the lithium line while nep 4780 shows a prominent line. The spectra have been continuum subtracted and offset on the vertical axis.

deviation of errors, which were typically not more than a few percent $(\approx 3 \mathrm{~mA})$.

For stars with $4000 \mathrm{~K} \leq T_{\text {eff }} \leq 6500 \mathrm{~K}$, the Li abundances $N(\mathrm{Li})$ were derived using the measured EWs and from a linear interpolation of the growth curves of Soderblom et al. (1993). Effective temperatures were derived from the spectral type (Allen 1973), as determined in Paper I; we derived the error in two subtypes from the published spectral types (Paper I), and this uncertainty was propagated into the $\mathrm{Li}$ abundances.

The lithium EW for binary stars was computed in relation to the combined continuum, which was the sum of continuum contributions from both components. Without information about the individual spectral types of both components, we were unable to separate the continuum contributions and obtain individual estimates of the lithium EW of each star (apart from the SB2 nep 5520). We measured the EWs for the suspected binary stars, but we emphasize that these measurements correspond to the unsolved blend including the contribution of both stars; we can therefore only state that these stars have lithium. While this is the best one can do with the available information, this procedure is likely to introduce additional errors. Therefore in Fig. 7, in which we report the lithium EWs measured for the subsample of single stars, such system are flagged with a different symbol (filled squares, while the EWs for the two components of nep 5520 are indicated as " $x$ ").

In Table A.2, we indicate the $\mathrm{Li}$ abundances $N(\mathrm{Li})$ derived, together with the NLTE corrections interpolated from the grid of Carlsson et al. (1994). When no lithium line was visible in the spectrum, we used the statistical noise measured from the spectral continuum, to determine an upper limit to the Li line EW, except for those stars with such high rotational velocity, that the lines were completely smeared out. To set constraints on the age of our targets, the lithium EWs of the Pleiades $\left(10^{8} \mathrm{yrs}\right)$ and Hyades $\left(10^{9}\right.$ yrs $)$ clusters are plotted in Fig. 7 for comparison (Soderblom et al. 1993, and 1990, respectively).

The comparison of the three samples of stars shows that the EWs of most targets lie between those of stars of similar temperature in the two clusters, and in a few cases are lower than those of the Hyades. 


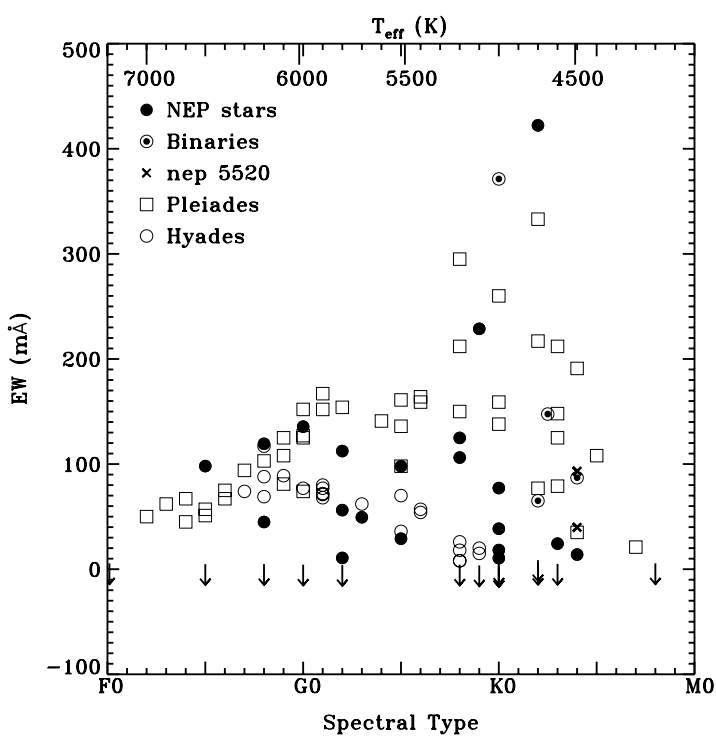

Fig. 7. Distribution of lithium equivalent widths of single NEP stars (filled dots) and binary stars (ringed dots; " $X$ " for nep 5520), with respect to spectral type, compared to Pleiades (empty squares) and Hyades (empty dots) distributions. Arrows indicate EW upper limits for those stars in which no lithium line was visible in the spectrum.

\section{Discussion}

We derive both radial and rotational velocities, and lithium abundances, for the stellar population of the NEP survey.

We use different methods to determine the same parameters, which enables us to infer the reliability of the analysis techniques employed. We obtain results that are consistent with each other, which demonstrated the robustness of our analysis. We quote the results in Table A.2: Col. 1 identifies stars with NEP numbers; Cols. 2, 3 provide the heliocentric $R V$ and the $v \sin i$ values, with estimated errors (we indicate that $3.5 \mathrm{~km} \mathrm{~s}^{-1}$ is the upper limit for measurements of rotational velocity that are below the instrumental profile width, as discussed in Sect. 3.2); Cols. 4-7 indicate the equivalent widths of the lithium line (6707.8 $\AA$ ), the temperature and its associated error (derived from spectral type), and lithium abundances (derived from the interpolation of the growth curves of Soderblom et al. 1993), respectively; Col. 8 reports the spectral types and in Col. 9 we indicate whether the star is a binary (label "x") or a double-line spectroscopic binary (label "SB2"). Label "*” indicates that we lack the Thorium-Argon lamp exposures for the nights in which data for these stars were acquired. These observations were wavelength-calibrated using lamp exposures acquired for the night closest in time. We assumed the same spectral type and temperature for the two component of SB2 nep 5520; this is an "ad hoc" assumption, which is reasonable because the lines are similar. In Table A.2, we indicate our poor knowledge about binaries with the label "?", close to the spectral type indication.

\subsection{Radial velocity}

In Fig. 2, we show the histogram of the heliocentric radial velocities, and superimpose a Gaussian radial velocity distribution for the target stars, adopting a standard deviation of $13 \mathrm{~km} \mathrm{~s}^{-1}$, that corresponds to the histogram variance, and a mean value of $-13.5 \mathrm{~km} \mathrm{~s}^{-1}$, that agrees well with the bulk of the data.
Table 3. Properties of high radial velocity stars.

\begin{tabular}{rcccc}
\hline \hline nep & Sp.type & $\begin{array}{c}\text { High } V_{\text {rad }} \\
\left(\geq|30| \mathrm{km} \mathrm{s}^{-1}\right)\end{array}$ & $\begin{array}{c}\text { High } V_{\text {rot }} \\
\left(\geq 15 \mathrm{~km} \mathrm{~s}^{-1}\right)\end{array}$ & Li \\
\hline 1690 & G2 & $-37.0 \pm 2.7$ & - & - \\
1800 & F5 & $-58.0 \pm 4.1$ & $16.3 \pm 1.4$ & $3.30 \pm 0.20$ \\
3560 & G8 & $-26.0 \pm 1.3$ & - & $2.30 \pm 0.30$ \\
$* 4090$ & G2 & $-41.6 \pm 2.2$ & - & - \\
4810 & F5 & $-37.0 \pm 1.6$ & - & - \\
5510 & K0 & $-53.5 \pm 3.0$ & $20.4 \pm 2.1$ & $3.70 \pm 0.50$ \\
\hline
\end{tabular}

*: see Note in Table A.1.

The Sun moves relative to the Local Standard of Rest (LSR) with a mean velocity of $\left|v_{\mathrm{LSR}}\right|=13.4 \mathrm{~km} \mathrm{~s}^{-1}$ (Binney \& Merrifield 1998).

The Gaussian radial velocity distribution, which represents a good fit for most of the targets reflects the motion of the Sun relative to the LSR. The distribution of radial velocity is consistent with that of a young population of disk stars of age $4 \times 10^{9} \mathrm{yrs}$ (Wilson 1963; Kraft 1967; Skumanich 1972; Simon 1985), or younger. We detect a small number (6) of stars with large radial velocity $\left(\geq|30| \mathrm{km} \mathrm{s}^{-1}\right)$. Nevertheless we cannot consider these stars as a statistical meaningful sample, because only 4 of these stars are within $2 \sigma$ from the mean value, one is within $3 \sigma$ and only one has a velocity which falls beyond $3 \sigma$ from the mean value with an extremely small probability $(|P|>3 \sigma \approx 0.3 \%)$.

In Table 3, we report the properties of these stars and the lithium abundances. In particular we want to know if these stars are older than the remainder of the observed stars or if they are peculiar in some way. Of these six stars, three of them (nep 1690, nep 4090, nep 4810) are slow rotators $\left(\leq 15 \mathrm{~km} \mathrm{~s}^{-1}\right)$ that have no detected $6708 \AA$ lithium line. These could be old stars. One of these stars (nep 3560) is a slow rotator and a Li line is detectable in its spectrum, and finally, two stars (nep 1800, nep 5510) are fast rotators $\left(\geq 15 \mathrm{~km} \mathrm{~s}^{-1}\right)$ and have measurable Li line in their spectra.

A possible explanation for the "high velocity" stars can be given in terms of binarity. Nep 5510 is probably a binary system. Analyses of spectra acquired for all other stars do not, however, confirm conclusively the presence of binarity.

Abundance analyses, in particular the measurement of the metallicity and chemical abundance ratios of several elements $([\alpha / \mathrm{Fe}],[\mathrm{O} / \mathrm{Fe}])$, could provide insight into the stellar populations, because abundances are a key indicator of which population a star belongs to.

\subsection{Rotational velocity}

Figure 4 shows that almost all stars have projected rotational velocities, which are interpreted as lower limits to actual values, that are comparable to or higher than that of the $\operatorname{Sun}\left(\approx 2 \mathrm{~km} \mathrm{~s}^{-1}\right)$.

Rotational velocity decreases with increasing age. Since magnetic activity depends on rotation and differential rotation, the level of magnetic activity and hence X-ray emission will be different for fast and slow rotators.

Our sample seems to be dominated by relatively young or intermediate-age stars. This statement is supported by Fig. 8, which shows the $v \sin i$ distribution as a function of the spectral type and compares it to the average trends for the Pleiades, Hyades and field stars; we indicate stars that have $\mathrm{NaI}\left(D_{1}, D_{2}\right)$ emission with square symbols, $\mathrm{H} \alpha$ emission with $\times$ symbols, and stars that have both $\mathrm{H} \alpha$ and $\mathrm{NaI}\left(D_{1}, D_{2}\right)$ emission with diamond symbols. We see that most stars have measurements 


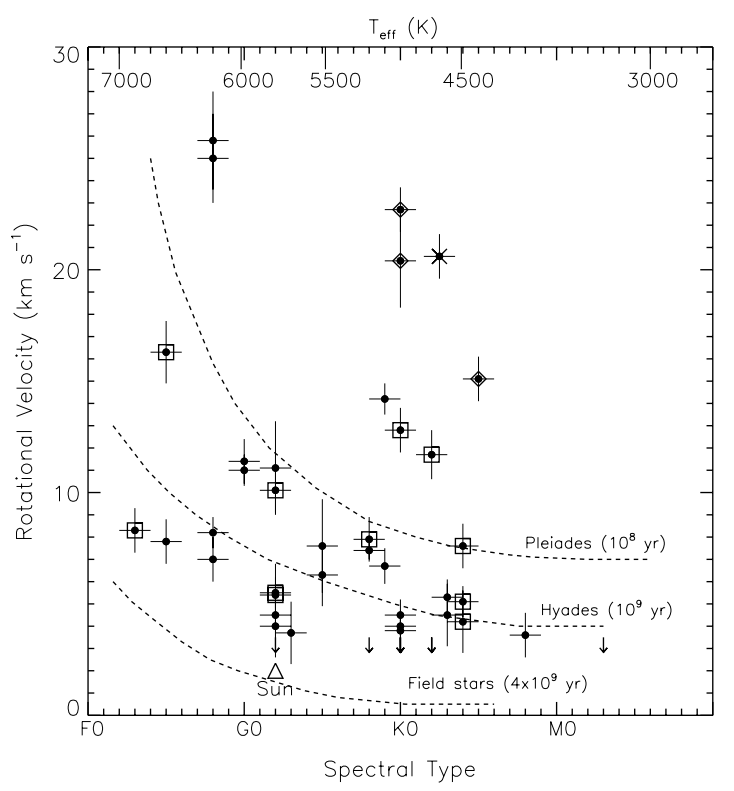

Fig. 8. Rotational velocity distribution with respect to spectral type (temperature); average trends of rotational velocity of Pleiades, Hyades and field stars, are shown (Favata et al. 1995; Kraft 1967; Soderblom 1983a,b; Skumanich 1972). With squares we indicate stars that have $\mathrm{Na} \mathrm{I}\left(D_{1}, D_{2}\right)$ emission; " $\times$ " indicates the presence of $\mathrm{H} \alpha$ emission and diamonds are for stars that have both $\mathrm{H} \alpha$ and $\mathrm{Na} \mathrm{I}\left(D_{1}, D_{2}\right)$ emission.

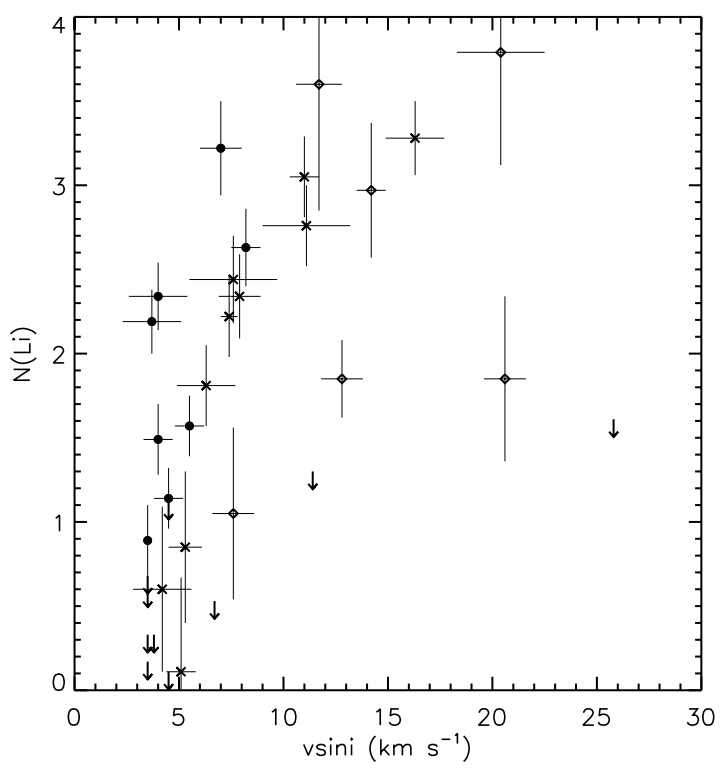

Fig. 9. Lithium abundances as a function of the rotational velocity: diamonds indicate stars with $v \sin i$ greater than those of Pleiades; " $x$ " indicate $v \sin i$ values between the average curves of Pleiades and Hyades; dots indicate $v \sin i$ values between the average curves of Hyades and field stars; down-pointing arrows indicate upper limits of lithium abundances.

that are consistent with an age between $10^{8}$ yrs (Pleiades age) and $4 \times 10^{9}$ yrs (field stars), compared to the age of the Sun, $5 \times 10^{9}$ yrs.

A small number of $\mathrm{K}$ stars have measured rotational velocities that are higher than measurements for the Pleiades. We propose that these stars have ages that are comparable to those of the Pleiades, or are even younger. Two of the stars could be binaries. For the remainder of the sample the youthness is the most plausible explanation.

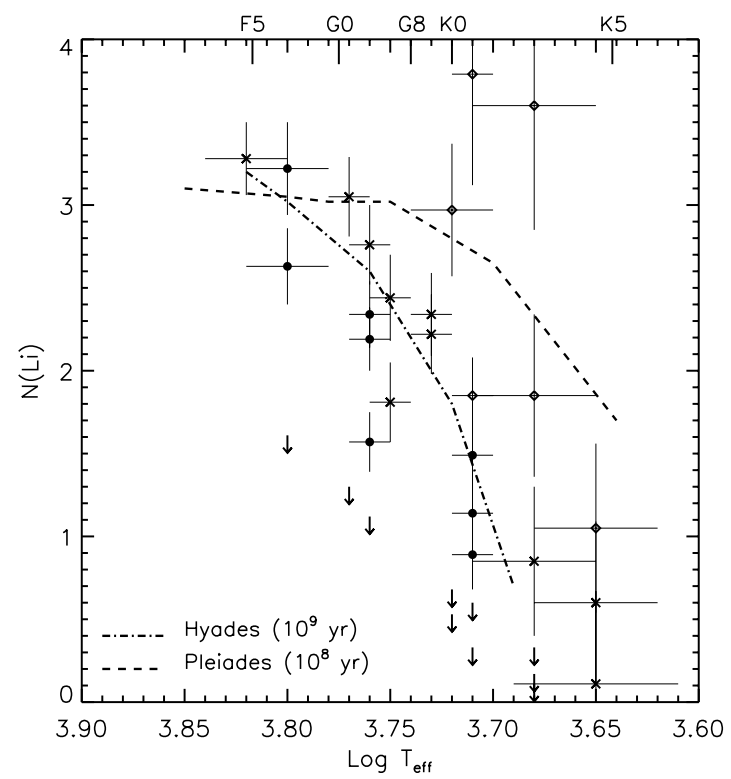

Fig. 10. Lithium abundances as a function of the effective temperatures: symbols as in Fig. 9. We indicate Pleiades and Hyades fits, obtained by Favata et al. (1993).

Table 4. Summary of X-ray source counts predictions for each spectral type and range of age derived from XCOUNT (from Paper I).

\begin{tabular}{lcccc}
\hline \hline Sp.type & $N$ (young) & $N$ (intermed.) & $N($ old $)$ & Total \\
\hline A & 0.63 & 1.41 & 0.28 & 2.32 \\
F0-F5 & 0.32 & 1.53 & 7.34 & 9.19 \\
F6-F9+G & 1.91 & 8.42 & 7.63 & 17.96 \\
K & 4.24 & 8.86 & 20.26 & 33.36 \\
\hline & 7.1 & 20.22 & 35.51 & 62.83 \\
\hline
\end{tabular}

Widely-separated binaries behave in a similar way to single stars because tidal forces can be neglected (Slettebak 1963; Weiss 1974). Close binaries, however, show altered rotation. Close binaries, of early spectral type, rotate more slowly than single stars, while those of later type rotate much faster than their single counterparts (Gray 1976, 1984).

In Figs. 9 and 10, we report the LTE lithium abundances, upper limits included, as a function of rotational velocity and temperature, respectively. Stars that show high lithium abundances and low rotation are probably young; this hypothesis is reinforced by the presence of emission in the $\mathrm{H} \alpha$ and $\mathrm{Na}$ I $D_{1}$ and $D_{2}$ lines, in most spectra (see Table A.3).

\subsection{Comparison with model results}

The predictions of the model XCOUNT (Favata et al. 1992; Micela et al. 1993) for the complete NEP survey, for each spectral type and age range are summarized in Table 4.

XCOUNT assumes an exponential disk-like spatial distribution of the stars, with a radial scale length of $3.5 \mathrm{kpc}$, as in Bahcall \& Soneira (1980). We followed the modifications of the Bahcall \& Soneira (1980) model of Micela et al. (1993), which introduced an age-dependent scale height, dividing stars into three age ranges: $10^{7}-10^{8}, 10^{8}-10^{9}$, and $10^{9}-10^{10}$ years, with scale heights of 100,200 and $400 \mathrm{pc}$, respectively. Micela et al. (1993) assumed X-ray luminosity functions derived from ROSAT observations of the Pleiades 
Table 5. Summary of F6-F9, G and K observations derived in this work vs. scaled predictions derived from XCOUNT, for each range of age. For 8 stars of our sample of late-F to G and K stars we did not measure lithium EWs (upper limits) due to the low $S / N$ ratio of their spectra and/or high rotation.

\begin{tabular}{lcccccccc}
\hline \hline Sp.type & \multicolumn{2}{c}{$N$ (young) } & \multicolumn{2}{c}{$N$ (intermed.) } & \multicolumn{2}{c}{$N($ old $)$} & \multicolumn{2}{c}{ Total } \\
\hline & pred. & obs. & pred. & obs. & pred. & obs. & pred. & obs. \\
\hline F6-F9+G & 1.15 & $7(9)$ & 5.05 & $4(5)$ & 4.58 & $6(8)$ & 10.78 & $17(22)$ \\
K & 1.70 & $7(10)$ & 3.54 & $5(5)$ & 8.10 & $7(7)$ & 13.34 & $19(22)$ \\
\hline & 2.85 & $14(19)$ & 8.59 & $9(10)$ & 12.68 & $13(15)$ & 24.12 & $36(44)$ \\
\hline
\end{tabular}

(Micela et al. 1996) and Hyades (Stern et al. 1995), and from Einstein data for nearby stars (Schmitt et al. 1985; Maggio et al. 1987; Barbera et al. 1993), which are considered to be prototypes for the three age ranges.

We scaled the XCOUNT predictions by a factor corresponding to the fraction of the stars that we observed $(\approx 0.6$ for late-F to $\mathrm{G}$ stars and $\approx 0.4$ for $\mathrm{K}$ stars), and assigned each star to a given age range based on its lithium EWs and/or rotational velocities. We used lithium as a primary indicator and assigned a star: to the $10^{7}-10^{8}$ years age range, if its lithium EWs were greater than (or comparable to) those of the Pleiades; to the $10^{8}-10^{9}$ years age range, if its lithium EWs were between the measurements shown by the average curves of Pleiades and Hyades; and to the $10^{9}-10^{10}$ years age range, if its lithium EWs were less than those of the Hyades. For those stars for which lithium is not detected, we used rotational velocities as an alternative indicator, applying the same criteria as for the lithium EWs. We focus on late-F to G and $\mathrm{K}$ type stars because our age indicators (lithium and rotation) are not appropriate for early-type stars. For late-F to $\mathrm{G}$ stars, we expect to find about 1.2 stars that have an age $\leq 10^{8} \mathrm{yrs}$, but instead find 7 stars. Similarly, we expect about 5 stars to have an intermediate value of age in the range $10^{8}-10^{9}$ years, and indeed we find 4 stars. For the age range $10^{9}-10^{10}$ years, we find 6 stars in contrast to about 5 predicted by the model. For K stars, we find 7 stars that have an age $\leq 10^{8}$ yrs, compared with about 2 stars predicted by the model; 5 stars to have an intermediate age, between $10^{8}-10^{9}$ years, with respect to about 4 predicted stars; and finally 7 stars in the range between $10^{9}-10^{10}$ years, in contrast to a prediction of 8 stars. Our results are summarized in Table 5: we indicate the observed and predicted (by XCOUNT model) stars for our sample, and, between brackets, the total counts of stars that may have an age in the range considered, on the basis of rotational velocities and lithium EWs, including those stars with only rotational velocity and no lithium measurements. For 8 of the late-F to $\mathrm{G}$ and $\mathrm{K}$ stars observed, we did not measure the upper limits of lithium EWs due to the low $S / N$ ratio of the spectra and/or high rotation.

The observed excess seems to be concentrated at young age. Our comparison suggests a burst of star formation, with an increasing of new-born stars by a factor of $\approx 4.5$ (or $\approx 3.5$ if we exclude 4 probable binary systems, concentrated at young age) in the last $10^{8}$ years, while it is consistent with a constant stellar birthrate in the intermediate and old ages, between $10^{8}-10^{10}$ years.

\section{Conclusions}

We derived fundamental quantities, such as radial and rotational velocities and lithium abundances, for stars detected in the NEP $\mathrm{X}$-ray survey. In Paper I, we confirmed the presence of an excess of yellow stars in the NEP sample and suggested that such an excess could be due to the presence of a young population, formed by a recent event of star formation. Moreover, there was no evidence for an equivalent excess of $\mathrm{M}$ stars, which would be necessary to identify the population as really young. We refer the reader to Paper I for a possible explanation of the absence of an excess. In the present work, $\mathrm{M}$ stars are poorly sampled $(\approx 0.05 \%$ of the entire sample of NEP M stars) and, as a consequence, we cannot state anything about their mean properties. Our results, which refer to $\mathrm{F}, \mathrm{G}$ and $\mathrm{K}$ stars, confirm that most of the stars seem to belong to a young age population. The population excess of the model prediction described in Paper I appears to be composed of mainly young stars (with ages comparable to those of Pleiades).

Our data suggest a burst in the birthrate, by a factor of $\approx 4$, in the last $10^{8}$ years. The Gould Belt is an asymmetry in the apparent distribution of the brightest stars in the sky, with respect to Galactic equator, probably associated with a recent star formation episode close to the Sun (Guillout et al. 1998). The NEP surveyed area is far from the Gould Belt and this star formation episode was not accounted for in the XCOUNT model, and is possible that other local, less prominent events of star formation occurred in the solar neighborhood. Nevertheless few stars, among the fastest $\mathrm{K}$ rotators, may be old binary systems with tidally locked rotation.

Acknowledgements. Based on observations made with the Italian Telescopio Nazionale Galileo (TNG) operated on the island of La Palma by the Fundaciòn Galileo Galilei of the INAF (Istituto Nazionale di Astrofisica) at the Spanish Observatorio del Roque de los Muchachos of the Instituto de Astrofisica de Canarias. We acknowledge support by the Marie Curie Fellowship Contract No. MTKD-CT-2004-002769 and ASI-INAF I/088/06/0 Contract. This research has made use of the SIMBAD database, operated at CDS, Strasbourg, France, and the NASA's Astrophysics Data System Abstract Service. We wish to thank the referee M. Guedel for his careful reading of the manuscript and for his suggestions to improve the paper. We wish to thank Javier Lopez Santiago for fruitful discussions.

\section{Appendix A: Observing log for the NEP spectra and results}

In this section, we present the observing log for the observations of stars detected in NEP survey (hereafter NEP spectra) and the results derived during the present study.

In Table A.1, we report the observing log for the NEP spectra, including identification numbers, observation dates, right ascension, declination (Eq. (2000.0)), spectral types (as derived from Micela et al. 2007), and total observing times.

In Table A.2, we provide our measurements: heliocentric $R V$ and $v \sin i$ values, with estimated errors, equivalent widths of lithium line $(6707.8 \AA)$, temperatures with associated errors (derived from spectral type), and lithium abundances (derived from the interpolation of the growth curves of Soderblom et al. 1993).

In Table A.3, we report the presence of $\mathrm{H} \alpha$ and $\mathrm{Na} \mathrm{I}\left(D_{1}, D_{2}\right)$ emission, and of a measurable lithium line at $6707.8 \AA$. 
Table A.1. Observing Log for the NEP spectra. Scan numbers are from Gioia et al. (2003).

\begin{tabular}{|c|c|c|c|c|c|c|}
\hline Scan & Date & $\begin{array}{c}\text { RA } \\
(\mathrm{h} \mathrm{m} \mathrm{s})\end{array}$ & $\begin{array}{l}\text { Dec } \\
\circ, \prime \prime\end{array}$ & Spectral type & $t_{i}(\mathrm{~s})$ & $S / N$ \\
\hline${ }^{*} 20$ & 2004 Jun. 28 & 175244.8 & +670020 & G8 & 5400 & 70 \\
\hline 260 & 2004 Jun. 01 & 175828.3 & +672608 & $\mathrm{G} 2$ & 2400 & 50 \\
\hline 330 & 2004 Jun. 02 & 175923.6 & +660255 & G9 & 1800 & 70 \\
\hline 370 & 2004 May 25 & 180002.2 & +664553 & F8 & 2400 & 90 \\
\hline${ }^{*} 1420$ & 2004 Mar. 08 & 171928.8 & +652229 & K0 & 3600 & 60 \\
\hline${ }^{*} 1441$ & 2004 Jan. 31 & 172005.6 & +620622 & $\mathrm{G} 2$ & 1200 & 100 \\
\hline 1470 & 2004 Mar. 09 & 172026.7 & +670337 & G8 & 1800 & 80 \\
\hline 1500 & 2004 Apr. 07 & 172110.9 & +694802 & $\mathrm{G} 2$ & 600 & 200 \\
\hline 1511 & 2004 Feb. 03 & 172142.5 & +620032 & K4 & 3600 & 60 \\
\hline 1580 & 2004 Apr. 07 & 172400.4 & +694030 & $\mathrm{K} 2$ & 3600 & 50 \\
\hline${ }^{*} 1600$ & 2004 Mar. 08 & 172426.8 & +641223 & G2 & 1800 & 50 \\
\hline 1601 & 2004 Mar. 09 & 172438.8 & +644051 & G0 & 1800 & 80 \\
\hline 1690 & 2004 Mar. 09 & 172645.4 & +693753 & G2 & 2400 & 60 \\
\hline 1800 & 2004 May 06 & 172939.4 & +684738 & F5 & 1200 & 110 \\
\hline 1840 & 2004 May 25 & 173019.9 & +695527 & G0 & 1800 & 90 \\
\hline 1960 & 2004 Apr. 07 & 173316.9 & +671208 & $\mathrm{F} 2$ & 600 & 170 \\
\hline 2050 & 2004 Mar. 09 & 173614.1 & +650227 & K0 & 2400 & 70 \\
\hline 2100 & 2004 May 06 & 173626.6 & +682037 & M3 & 600 & 170 \\
\hline 2210 & 2004 Jun. 02 & 173916.1 & +702009 & F8 & 1200 & 80 \\
\hline 2250 & 2004 Mar. 08 & 173956.1 & +650004 & K0 & 2400 & 200 \\
\hline 2360 & 2004 Jun. 01 & 174226.5 & +690758 & F8 & 1200 & 120 \\
\hline 2400 & 2004 Mar. 09 & 174301.6 & +660646 & G2 & 1800 & 90 \\
\hline 2470 & 2004 Jun. 02 & 174351.7 & +703139 & F3 & 1800 & 40 \\
\hline 2480 & 2004 Jun. 01 & 174400.6 & +701527 & K0 & 2400 & 50 \\
\hline *2970 & 2004 Jan. 31 & 174903.9 & +624748 & $\mathrm{~F} 2$ & 600 & 290 \\
\hline$* 3080$ & 2004 Jun. 28 & 175025.3 & +704536 & $\mathrm{~K} 2.5$ & 1800 & 90 \\
\hline 3220 & 2004 Jun. 01 & 175256.0 & +662510 & K0 & 600 & 150 \\
\hline 3560 & 2004 Jun. 28 & 175703.7 & +684914 & G8 & 1800 & 110 \\
\hline 3610 & 2004 May 06 & 175801.4 & +640934 & K4 & 2700 & 70 \\
\hline 3690 & 2004 May 06 & 175848.0 & +635039 & A2 & 1200 & 120 \\
\hline 3710 & 2004 Jun. 30 & 175913.8 & +640833 & $\mathrm{F} 2$ & 1200 & 200 \\
\hline$* 4090$ & 2004 Jun. 28 & 180530.5 & +694517 & G2 & 2400 & 50 \\
\hline 4100 & 2004 May 25 & 180530.3 & +621903 & K0 & 1200 & 150 \\
\hline 4130 & 2004 Jun. 02 & 180544.8 & +655158 & F5 & 1200 & 80 \\
\hline 4180 & 2004 May 25 & 180641.0 & +641318 & K0 & 600 & 440 \\
\hline 4380 & 2004 Jun. 01 & 180845.4 & +625637 & G5 & 1200 & 120 \\
\hline 4470 & 2004 Jun. 29 & 180955.8 & +694039 & K3 & 1200 & 160 \\
\hline 4530 & 2004 Jun. 29 & 181049.9 & +701609 & G9 & 4800 & 80 \\
\hline 4780 & 2004 Jun. 29 & 181348.6 & +683132 & $\mathrm{K} 2$ & 2400 & 80 \\
\hline 4810 & 2004 Jun. 03 & 181351.4 & +642357 & F5 & 180 & 300 \\
\hline *4931 & 2004 Jun. 28 & 181621.2 & +652939 & K8 & 1200 & 170 \\
\hline 4970 & 2004 Jun. 03 & 181649.7 & +650426 & $\mathrm{~K} 2$ & 2400 & 60 \\
\hline 5060 & 2004 Jun. 29 & 181831.9 & +704217 & G5 & 1800 & 90 \\
\hline 5220 & 2004 Jun. 03 & 182019.2 & +651919 & F8 & 600 & 120 \\
\hline *5320 & 2004 Jun. 28 & 182146.8 & +635710 & K0 & 1800 & 90 \\
\hline 5510 & 2004 Jun. 03 & 182509.0 & +645021 & K0 & 600 & 260 \\
\hline 5520 & 2004 Jun. 03 & 182532.9 & +623415 & K4 & 2400 & 60 \\
\hline 5950 & 2004 Jun. 30 & 183229.5 & +683652 & G0 & 600 & 190 \\
\hline 6030 & 2004 Jun. 29 & 183347.8 & +651333 & K3 & 1200 & 150 \\
\hline 6051 & 2004 Jun. 29 & 183433.6 & +693145 & K5 & 3600 & 50 \\
\hline 6160 & 2004 Jun. 30 & 183613.5 & +652915 & F0 & 300 & 230 \\
\hline 6163 & 2004 Jun. 29 & 183622.8 & +665454 & G3 & 600 & 180 \\
\hline 6350 & 2004 Jun. 29 & 183925.4 & +690254 & M3 & 2400 & 60 \\
\hline 6400 & 2004 Jun. 29 & 184044.4 & +703847 & $\mathrm{~K} 2$ & 1200 & 70 \\
\hline 6410 & 2004 Jun. 30 & 184056.6 & +624454 & K0 & 300 & 300 \\
\hline 6510 & 2004 Jun. 29 & 184312.6 & +695554 & F0 & 1200 & 80 \\
\hline
\end{tabular}

* We lack the thorium-argon lamp exposures for the nights in which these stars were acquired. They were wavelength calibrated using lamp exposures of the nearest night. 
Table A.2. Results: $V_{\text {rad }}, V_{\text {rot }}$, Li equivalent width, Li abundance, Binarity.

\begin{tabular}{|c|c|c|c|c|c|c|c|c|}
\hline nep & $V_{\text {rad }}\left(\mathrm{km} \mathrm{s}^{-1}\right)$ & $V_{\text {rot }}\left(\mathrm{km} \mathrm{s}^{-1}\right)$ & $E W_{\mathrm{Li}}(\mathrm{m} \AA)$ & $T_{\text {eff }}(\mathrm{K})$ & $N_{\mathrm{Li}}$ & $N_{\mathrm{Li}}^{\mathrm{NLTE}}$ & Spectral type & notes \\
\hline${ }^{*} 20$ & $-17.3 \pm 0.9$ & $\leq 3.5$ & $<4.3$ & $5310 \pm 150$ & $<0.68$ & $<0.77$ & G8 & \\
\hline 260 & $-19.5 \pm 1.1$ & $11.1 \pm 2.1$ & 112.4 & $5790 \pm 150$ & $2.76 \pm 0.24$ & $2.71 \pm 0.19$ & $\mathrm{G} 2$ & \\
\hline 330 & $-35.4 \pm 1.0$ & $6.7 \pm 0.8$ & $<3.6$ & $5250 \pm 150$ & $<0.53$ & & G9 & \\
\hline 370 & $-15.7 \pm 1.3$ & $7.0 \pm 1.0$ & 119.3 & $6250 \pm 300$ & $3.22 \pm 0.28$ & $3.09 \pm 0.20$ & F8 & \\
\hline${ }^{*} 1420$ & & $22.7 \pm 1.0$ & - & $5150 \pm 150$ & & & K0 & \\
\hline${ }^{*} 1441$ & $-15.7 \pm 1.5$ & $4.0 \pm 1.4$ & 56.2 & $5790 \pm 150$ & $2.34 \pm 0.20$ & $2.34 \pm 0.18$ & $\mathrm{G} 2$ & \\
\hline 1470 & $-10.2 \pm 1.0$ & $7.4 \pm 0.4$ & 106.2 & $5310 \pm 150$ & $2.22 \pm 0.24$ & $2.27 \pm 0.21$ & G8 & \\
\hline 1500 & $-17.0 \pm 0.8$ & $5.5 \pm 0.7$ & 10.7 & $5790 \pm 150$ & $1.57 \pm 0.18$ & $1.59 \pm 0.18$ & G2 & \\
\hline 1511 & & & 87.0 & $4450 \pm 350$ & $1.01 \pm 0.50$ & & K4 (?) & $\mathrm{x}$ \\
\hline 1580 & & & 65.1 & $4830 \pm 300$ & $1.36 \pm 0.40$ & $1.55 \pm 0.37$ & K2 (?) & $\mathrm{x}$ \\
\hline$* 1600$ & $-18.8 \pm 0.9$ & $5.4 \pm 1.4$ & - & $5790 \pm 150$ & & & $\mathrm{G} 2$ & \\
\hline 1601 & $-13.0 \pm 1.4$ & $11.0 \pm 0.7$ & 135.6 & $5940 \pm 150$ & $3.05 \pm 0.24$ & $2.94 \pm 0.18$ & G0 & \\
\hline 1690 & $-37.0 \pm 2.7$ & $10.1 \pm 1.0$ & - & $5790 \pm 150$ & & & $\mathrm{G} 2$ & \\
\hline 1800 & $-58.0 \pm 4.1$ & $16.3 \pm 1.4$ & 98.1 & $6650 \pm 300$ & $3.28 \pm 0.22$ & $3.16 \pm 0.18$ & F5 & \\
\hline 1840 & & $\geq 30.0$ & - & $5940 \pm 150$ & & & G0 & \\
\hline 1960 & & $\geq 50.0$ & - & $7000 \pm 300$ & & & $\mathrm{~F} 2$ & \\
\hline 2050 & $-7.6 \pm 1.8$ & $12.8 \pm 1.0$ & 77.2 & $5150 \pm 150$ & $1.85 \pm 0.23$ & $1.96 \pm 0.21$ & K0 & \\
\hline 2100 & & & - & $3500 \pm 350$ & & & M3 & \\
\hline 2210 & $-7.1 \pm 1.8$ & $25.0 \pm 2.0$ & - & $6250 \pm 300$ & & & F8 (?) & $\mathrm{x}$ \\
\hline 2250 & $-7.4 \pm 0.9$ & $3.8 \pm 0.7$ & $<3.0$ & $5150 \pm 150$ & $<0.33$ & & K0 & \\
\hline 2360 & $-26.2 \pm 1.4$ & $8.2 \pm 0.7$ & 44.9 & $6250 \pm 300$ & $2.63 \pm 0.23$ & $2.58 \pm 0.16$ & F8 & \\
\hline 2400 & $-14.3 \pm 0.9$ & $4.5 \pm 1.4$ & $<3.9$ & $5790 \pm 150$ & $<1.12$ & $<1.16$ & G2 & \\
\hline 2470 & & $8.3 \pm 1.0$ & - & $6950 \pm 300$ & & & F3 & \\
\hline 2480 & & $\geq 40.0$ & - & $5150 \pm 150$ & & & K0 & \\
\hline *2970 & & $\geq 60.0$ & - & $7000 \pm 300$ & & & F2 (?) & $\mathrm{x}$ \\
\hline *3080 & $-12.9 \pm 1.7$ & $20.6 \pm 1.0$ & 147.6 & $4800 \pm 350$ & $1.85 \pm 0.49$ & $2.00 \pm 0.53$ & $\mathrm{~K} 2.5(?)$ & $\mathrm{x}$ \\
\hline 3220 & $-25.1 \pm 1.0$ & $\leq 3.5$ & $<5.4$ & $5150 \pm 150$ & $<0.60$ & $<0.72$ & K0 & \\
\hline 3560 & $-2.6 \pm 1.3$ & $7.9 \pm 1.0$ & 124.9 & $5310 \pm 150$ & $2.34 \pm 0.25$ & $2.38 \pm 0.24$ & G8 & \\
\hline 3610 & $+9.7 \pm 1.0$ & $5.1 \pm 0.7$ & 13.9 & $4450 \pm 400$ & $0.11 \pm 0.56$ & & K4 & \\
\hline 3690 & & & - & $9000 \pm 800$ & & & $\mathrm{~A} 2$ & \\
\hline 3710 & & $\geq 30.0$ & - & $7000 \pm 300$ & & & $\mathrm{~F} 2$ & \\
\hline *4090 & $-40.7 \pm 2.2$ & $\leq 3.5$ & - & $5790 \pm 150$ & & & G2 & \\
\hline 4100 & $+10.0 \pm 0.9$ & $4.5 \pm 0.7$ & 18.2 & $5150 \pm 150$ & $1.14 \pm 0.18$ & $1.27 \pm 0.31$ & K0 & \\
\hline 4130 & & $\geq 40.0$ & - & $6650 \pm 300$ & & & F5 & \\
\hline 4180 & $-13.6 \pm 1.0$ & $\leq 3.5$ & $<3.0$ & $5150 \pm 150$ & $<0.33$ & & K0 & \\
\hline 4380 & $-21.8 \pm 1.1$ & $6.3 \pm 1.4$ & 29.0 & $5560 \pm 200$ & $1.81 \pm 0.24$ & $1.86 \pm 0.23$ & G5 & \\
\hline 4470 & $-13.5 \pm 0.8$ & $5.3 \pm 0.8$ & 24.3 & $4800 \pm 350$ & $0.85 \pm 0.45$ & $1.06 \pm 0.44$ & K3 & \\
\hline 4530 & $-21.6 \pm 1.8$ & $14.2 \pm 0.7$ & 228.7 & $5300 \pm 150$ & $2.97 \pm 0.40$ & $2.84 \pm 0.26$ & G9 & \\
\hline 4780 & $-26.3 \pm 2.1$ & $11.7 \pm 1.1$ & 422.3 & $4830 \pm 300$ & $3.60 \pm 0.75$ & $3.40 \pm 0.65$ & $\mathrm{~K} 2$ & \\
\hline 4810 & $-37.0 \pm 1.6$ & $7.8 \pm 1.0$ & - & $6650 \pm 300$ & & & F5 & \\
\hline *4931 & $+2.5 \pm 1.7$ & $3.6 \pm 1.0$ & $<5.5$ & $4000 \pm 150$ & $<-0.8$ & & K8 & \\
\hline 4970 & $+14.3 \pm 0.9$ & $\leq 3.5$ & $<8.6$ & $4830 \pm 300$ & $<0.41$ & & $\mathrm{~K} 2$ & \\
\hline 5060 & $-2.3 \pm 0.9$ & $7.6 \pm 2.1$ & 97.9 & $5560 \pm 200$ & $2.44 \pm 0.26$ & $2.45 \pm 0.22$ & G5 & \\
\hline 5220 & $-18.9 \pm 2.0$ & $25.8 \pm 2.2$ & $<4.8$ & $6250 \pm 300$ & $<1.61$ & $<1.59$ & F8 & \\
\hline *5320 & $-19.4 \pm 0.8$ & $4.0 \pm 0.7$ & 38.5 & $5150 \pm 150$ & $1.49 \pm 0.21$ & $1.62 \pm 0.20$ & K0 & \\
\hline 5510 & $-53.5 \pm 3.0$ & $20.4 \pm 2.1$ & 371.3 & $5150 \pm 150$ & $3.79 \pm 0.67$ & $3.46 \pm 0.54$ & K0 (?) & $\mathrm{x}$ \\
\hline $5520^{a}$ & $+8.0 \pm 1.3$ & $7.6 \pm 1.0$ & 93.2 & $4450 \pm 350$ & $1.05 \pm 0.51$ & & K4 (?) & SB2 \\
\hline $5520^{b}$ & $+25.7 \pm 2.3$ & $4.2 \pm 1.4$ & 39.8 & $4450 \pm 350$ & $0.60 \pm 0.49$ & & K4 (?) & \\
\hline 5950 & $-20.6 \pm 1.4$ & $11.4 \pm 1.0$ & $<4.3$ & $5940 \pm 150$ & $<1.30$ & $<1.31$ & G0 & \\
\hline 6030 & $-13.2 \pm 1.0$ & $4.5 \pm 1.4$ & $<5.0$ & $4780 \pm 350$ & $<0.11$ & & K3 & \\
\hline 6051 & $-9.1 \pm 1.3$ & $15.1 \pm 1.0$ & - & $4410 \pm 400$ & & & K5 & \\
\hline 6160 & & & - & $7300 \pm 300$ & & & F0 & \\
\hline 6163 & $-7.4 \pm 0.5$ & $3.7 \pm 1.4$ & 49.4 & $5700 \pm 150$ & $2.19 \pm 0.19$ & $2.21 \pm 0.18$ & G3 & \\
\hline 6350 & & $\leq 3.5$ & - & $3500 \pm 350$ & & & M3 & \\
\hline 6400 & $-23.9 \pm 0.8$ & $\leq 3.5$ & $<5.0$ & $4830 \pm 300$ & $<0.17$ & & $\mathrm{~K} 2$ & \\
\hline 6410 & $-25.6 \pm 1.2$ & $\leq 3.5$ & 10.5 & $5150 \pm 150$ & $0.89 \pm 0.21$ & $1.02 \pm 0.21$ & K0 & \\
\hline 6510 & & & - & $7300 \pm 300$ & & & F0 & \\
\hline
\end{tabular}

Note: an $\mathrm{x}$ in column eight indicates that the star is (or it is supposed to be) binary. We indicate SB2 for double-lined spectroscopic binary.

* We lack the thorium-argon lamp exposures for the nights in which these stars were acquired. They were wavelength calibrated using lamp exposures of the nearest night.

${ }^{a}$ Primary and ${ }^{b}$ secondary component of nep 5520 SB2 system. 
Table A.3. Presence of $\mathrm{H} \alpha$ and $\mathrm{Na} \mathrm{I}\left(D_{1}, D_{2}\right)$ emission and presence of lithium feature (6707.8 $\AA$ ).

\begin{tabular}{|c|c|c|c|c|}
\hline nep & binary & $\mathrm{H} \alpha$ & $\mathrm{Na}$ I $\left(D_{1}, D_{2}\right)$ & $\mathrm{Li} \mathrm{I}$ \\
\hline${ }^{*} 20$ & & - & - & - \\
\hline 260 & & - & - & $\mathrm{x}$ \\
\hline 330 & & - & - & - \\
\hline 370 & & - & - & $\mathrm{x}$ \\
\hline${ }^{*} 1420$ & & $\mathrm{x}$ & $\mathrm{x}$ & - \\
\hline *1441 & & - & - & $\mathrm{x}$ \\
\hline 1470 & & - & - & $\mathrm{x}$ \\
\hline 1500 & & - & $\mathrm{x}$ & $\mathrm{x}$ \\
\hline 1511 & $\mathrm{x}$ & - & $\mathrm{x}$ & $\mathrm{x}$ \\
\hline 1580 & $\mathrm{x}$ & $\mathrm{x}$ & $\mathrm{x}$ & $\mathrm{x}$ \\
\hline$* 1600$ & $\mathrm{x}$ & - & $\mathrm{x}$ & - \\
\hline 1601 & & - & - & $\mathrm{x}$ \\
\hline 1690 & & - & $\mathrm{x}$ & - \\
\hline 1800 & & - & $\mathrm{x}$ & $\mathrm{x}$ \\
\hline 1840 & & - & - & - \\
\hline 1960 & & - & - & - \\
\hline 2050 & & - & $\mathrm{x}$ & $\mathrm{x}$ \\
\hline 2100 & & - & - & - \\
\hline 2210 & $\mathrm{x}$ & - & - & - \\
\hline 2250 & & - & - & - \\
\hline 2360 & & - & - & $\mathrm{x}$ \\
\hline 2400 & & - & - & - \\
\hline 2470 & & - & $\mathrm{x}$ & - \\
\hline 2480 & & $\mathrm{x}$ & $\mathrm{x}$ & - \\
\hline *2970 & & - & - & - \\
\hline *3080 & $\mathrm{x}$ & $\mathrm{x}$ & - & $\mathrm{x}$ \\
\hline 3220 & & - & - & - \\
\hline 3560 & & - & $\mathrm{x}$ & $\mathrm{x}$ \\
\hline 3610 & & - & $\mathrm{x}$ & $\mathrm{x}$ \\
\hline 3690 & & - & - & - \\
\hline 3710 & & - & $\mathrm{x}$ & - \\
\hline *4090 & & $\mathrm{x}$ & $\mathrm{x}$ & - \\
\hline 4100 & & - & - & $\mathrm{x}$ \\
\hline 4130 & & - & - & - \\
\hline 4180 & & - & - & - \\
\hline 4380 & & - & - & $\mathrm{x}$ \\
\hline 4470 & & - & - & $\mathrm{x}$ \\
\hline 4530 & & - & - & $\mathrm{x}$ \\
\hline 4780 & & - & $\mathrm{x}$ & $\mathrm{x}$ \\
\hline 4810 & & - & - & - \\
\hline$* 4931$ & & - & - & - \\
\hline 4970 & & - & $\mathrm{x}$ & - \\
\hline 5060 & & - & - & $\mathrm{x}$ \\
\hline 5220 & & - & - & - \\
\hline *5320 & & - & - & $\mathrm{x}$ \\
\hline 5510 & $\mathrm{x}$ & $\mathrm{x}$ & $\mathrm{x}$ & $\mathrm{x}$ \\
\hline 5520 & SB2 & - & $\mathrm{x}$ & $\mathrm{x}$ \\
\hline 5950 & & - & - & - \\
\hline 6030 & & - & - & - \\
\hline 6051 & & $\mathrm{x}$ & $\mathrm{x}$ & - \\
\hline 6160 & & - & - & - \\
\hline 6163 & & - & - & $\mathrm{x}$ \\
\hline 6350 & & $\mathrm{x}$ & $\mathrm{x}$ & - \\
\hline 6400 & & - & - & - \\
\hline 6410 & & - & - & $\mathrm{x}$ \\
\hline 6510 & & - & - & - \\
\hline
\end{tabular}

* We lack the thorium-argon lamp exposures for the nights in which these stars were acquired. They were wavelength calibrated using lamp exposures of the nearest night. 


\section{References}

Allen, C. W. 1973 Astrophysical quantities (London: University of London, Athlone Press), 3rd edn.

Bahcall, J. N., \& Soneira, R. M. 1980, ApJS, 44, 73

Bailer-Jones, C. A. L. 2004, A\&A, 419, 703

Barbera, M., Micela, G., Sciortino, S., Harnden, F. R., Jr., \& Rosner, R. 1993, ApJ, 414, 846

Bensby, T., Feltzing, S., \& Lundström, I. 2004, A\&A, 415, 155

Binney, J., \& Merrifield, M. 1998, Galactic astronomy/James Binney and Michael Merrifield, Princeton series in astrophysics (Princeton, NJ: Princeton University Press)

Carlsson, M., Rutten, R. J., Bruls, J. H. M. J., \& Shchukina, N. G. 1994, A\&A, 288, 860

Dehnen, W., \& Binney, J. J. 1998, MNRAS, 298, 387

Della Ceca, R., Maccacaro, T., Caccianiga, A., et al. 2004, A\&A, 428, 383

ESA 1997, The Hipparcos and Thyco catalogues, ESA SP-1200

Favata, F., Sciortino, S., Rosner, R., \& Vaiana, G. S. 1988, ApJ, 324, 1010

Favata, F., Micela, G., Sciortino, S., \& Vaiana, G. S. 1992, A\&A, 256, 86

Favata, F., Barbera, M., Micela, G., \& Sciortino, S. 1993, A\&A, 277, 428

Favata, F., Barbera, M., Micela, G., \& Sciortino, S. 1995, A\&A, 295, 147

Feigelson, E. D., \& Montmerle, T. 1999, ARA\&A, 37, 363

Feigelson, E. D., Hornschemeier, A. E., Micela, G., et al. 2004, ApJ, 611, 1107

Fekel, F. C. 1997, PAsP, 109, 514

Gioia, I. M., Maccacaro, T., Schild, R. E., et al. 1990, ApJS, 72, 567

Gioia, I. M., Henry, J. P., Mullis, C. R., et al. 2001, ApJ, 553, L105

Gioia, I. M., Henry, J. P., Mullis, C. R., et al. 2003, ApJS, 149, 29

Gioia, I. M., Wolter, A., Mullis, C. R., et al. 2004, A\&A, 428, 867

Gray, D. F. 1976, Research supported by the National Research Council of Canada (New York: Wiley-Interscience), 484

Gray, D. F. 1984, ApJ, 281, 719

Guedel, M., Guinan, E. F., \& Skinner, S. L. 1997, ApJ, 483, 947

Guillout, P., Sterzik, M. F., Schmitt, J. H. M. M., et al. 1998, A\&A, 334, 540

Henry, J. P., Gioia, I. M., Mullis, C. R., et al. 2001, ApJ, 553, L109

Henry, J. P., Mullis, C. R., Voges, W., et al. 2006, ApJS, 162, 304

Johnson, D. R. H., \& Soderblom, D. R. 1987, AJ, 93, 864

Kraft, R. P. 1967, ApJ, 150, 551

Kurucz, R. L. 1993, ATLAS9 Stellar Atmosphere Programs and $2 \mathrm{~km} \mathrm{~s}^{-1}$ grid, Kurucz CD-ROM No. 13 (Cambridge, Mass.: Smithsonian Astrophysical Observatory)
Kurucz, R. L. 1995, Astrophysical Applications of Powerful New Databases, 78, 205

López-Santiago, J., Micela, G., Sciortino, S., et al. 2007, A\&A, 463, 165

Maggio, A., Sciortino, S., Vaiana, G. S., et al. 1987, ApJ, 315, 687

Micela, G., Sciortino, S., Serio, S., et al. 1985, ApJ, 292, 172

Micela, G., Sciortino, S., Vaiana, G. S., et al. 1988, ApJ, 325, 798

Micela, G., Sciortino, S., \& Favata, F. 1993, ApJ, 412, 618

Micela, G., Sciortino, S., Kashyap, V., Harnden, F. R., Jr., \& Rosner, R. 1996, ApJS, 102, 75

Micela, G., Sciortino, S., \& Favata, F. 2001, Young Stars Near Earth: Progress and Prospects, ASP Conf. Ser., 244, 177

Micela, G., Affer, L., Favata, F., et al. 2007, A\&A, 461, 97 (Paper I)

Mullis, C. R. 2001, Ph.D. Thesis

Mullis, C. R., McNamara, B. R., Quintana, H., et al. 2003, ApJ, 594, 154

Mullis, C. R., Henry, J. P., Gioia, I. M., et al. 2004a, ApJ, 617, 192

Mullis, C. R., Vikhlinin, A., Henry, J. P., et al. 2004b, ApJ, 607, 175

Pallavicini, R., Golub, L., Rosner, R., et al. 1981, ApJ, 248, 279

Rhode, K. L., Herbst, W., \& Mathieu, R. D. 2001, AJ, 122, 3258

Scheffler, H., \& Elsässer, H. 1988, in Physics of the Galaxy and Interstellar Matter (Berlin: Springer Verlag)

Schmitt, J. H. M. M., Golub, L., Harnden, F. R., Jr., et al. 1985, ApJ, 290, 307

Sciortino, S., Favata, F., \& Micela, G. 1995, A\&A, 296, 370

Slettebak, A. 1963, ApJ, 138, 118

Skumanich, A. 1972, ApJ, 171, 565

Sneden, C. A. 1973, Ph.D. Thesis (Austin: University of Texas)

Soderblom, D. R. 1982, ApJ, 263, 239

Soderblom, D. R. 1983, ApJ, 53, 1

Soderblom, D. R., Pendleton, J., \& Pallavicini, R. 1989, AJ, 97, 539

Soderblom, D. R., Oey, M. S., Johnson, D. R. H., \& Stone, R. P. S. 1990, AJ, 99, 595

Soderblom, D. R., Jones, B. F., BalaChandran, S., et al. 1993, AJ, 106, 1059

Stern, R. A., Schmitt, J. H. M. M., \& Kahabka, P. T. 1995, ApJ, 448, 683

Strassmeier, K. G., \& Fekel, F. C. 1990, A\&A, 230, 389

Tonry, J., \& Davis, M. 1979, AJ, 84, 1511

Voges, W., Henry, J. P., Briel, U. G., et al. 2001, ApJ, 553, L119

Weiss, N. O. 1974, Saas-Fee Advanced Course 4: Magnetohydrodynamics, 185 